\title{
POTENTIAL FIELD BASED INTEGRATED EXPLORATION FOR MULTI-ROBOT TEAMS
}

\author{
Miguel Juliá, Arturo Gil, Luis Payá and Óscar Reinoso \\ Miguel Hernández University, System Engineering Department \\ Avda. Universidad s/n. Edif. Torreblanca, 03202 Elche-Alicante, Spain \\ \{mjulia, arturo.gil, lpaya, o.reinoso\}@umh.es
}

Keywords: $\quad$ Integrated Exploration, SPLAM, Potential Fields, Cooperative Mobile Robotics

\begin{abstract}
In this paper we present an approach for multi-robot cooperative exploration based on the potential field generated by several basic behaviours. When an unknown environment is explored the uncertainty in the localization normally grows, this fact may cause the failure of the Simultaneous Localization and Mapping (SLAM) algorithm, and thus constructing an useless and inaccurate map. The exploration algorithm described here considers the current knowledge of the environment, the location of the robots and the uncertainty in their positions in order to return to previously explored areas when it is needed. These actions definitely help the SLAM algorithm to build a precise map. Several simulations are presented that demonstrate the validity of the approach.
\end{abstract}

\section{INTRODUCTION}

In the last years, a large number of applications have emerged that require the utilization of cooperative mobile robots. Most of these applications require the robot team to be able to explore unknown environments autonomously. Employing multiple robots instead of a single robot in exploration is an advantage because the exploration time could be reduced (Cao et al., 1995).

Simultaneous Localization and Mapping (SLAM) techniques are generally used to explore an unknown environment. They allow to build a map that describes the environment while simultaneously using that map to localize the robots. However, the result obtained by the SLAM algorithm strongly depends on the trajectories performed by the robots (Stachniss et al., 2005). When the robots travel through unknown environments, the uncertainty over their position increases and the construction of the map becomes difficult. Returning to previously explored areas or closing loops reduces the uncertainty over the pose of the robots and improves the SLAM process.

Typical exploration algorithms do not take localization uncertainty into account and direct the exploration in order to minimize the distance traveled while maximizing the information gained. However, the solution presented here explores the environment efficiently and also considers the requisites of the SLAM algorithm. Our algorithm considers returning to previously explored places when the uncertainty becomes too large. This idea has been previously exploited by other authors and is commonly denoted as Integrated Exploration or SPLAM (Simultaneous Planning Localization And Mapping). A solution to the SPLAM problem enables a mobile robot to acquire data from sensors by autonomously moving through its environment while at the same time building a map. The main contribution of this paper is a new technique for Integrated Exploration for multirobot teams.

The remainder of the paper is structured as follows. Section 2 discusses related work and Section 3 presents the behaviour based exploration algorithm. In Section 4 we explain the active localization state. Next, Section 5 presents simulation results to test the functionality of the method proposed. Finally, the main conclusions and future work are presented. 


\section{RELATED WORK}

Exploration techniques work basically using the frontier concept introduced by (Yamauchi, 1997). He divided the map into a regular grid of cells where to represent the occupation probability. At the beginning of the exploration all the cells are unknown, so they are initialized with an occupation probability of 0.5 . This value is updated with the information of the sensors of the robots during the exploration. Relying on the occupation probability for each cell, the cells are labeled as free, occupied or unknown. Frontier cells are free cells that lie next to an unknown cell.

A group of exploration methods employ path planning techniques in order to direct the robots to the frontier cells (Simmons et al., 2000; Burgard et al., 2005; Zlot et al., 2002). They differ in the coordination strategies used to assign a frontier to each robot: the robots can go to the nearest frontier (Yamauchi, 1997) or they can follow a cost-utility model to make their assignments. Normally, the cost is the length of the path to a frontier cell, whereas utility could be understood in different ways: (Simmons et al., 2000) consider the utility as the expected visible area behind the frontier. (Burgard et al., 2005) consider in the utility function the proximity of frontiers assigned to other robots. (Zlot et al., 2002) suggest using a market economy where the robots negotiate their assignments.

Another group of exploration techniques makes use of potential field methods (Arkin and Diaz, 2002). Potential field based exploring methods take into account a set of behaviours to generate a resultant potential field. The most common behaviours in exploration are attraction to frontiers and repulsion from obstacles and other robots. This leads to the avoidance of other robots and collisions and also improves the exploration by dispersing the robots. As stated by many authors, the main drawback of this technique is the occurrence of local minima in the potential field, which may trap the robot and block the exploration process. A common solution to this problem is to plan a path to a frontier cell in order to get the robot out from the local minimum (Lau, 2003).

A few authors used integrated exploration in the last years (Feder et al., 1999; Bourgoult et al., 2002; Makarenko et al., 2002; Sim et al., 2004; Stachniss et al., 2005). (Feder et al., 1999) decide the next movement for robots by optimizing the information gain of the environment and minimizing the uncertainty in the localization of the robot. (Bourgoult et al., 2002) and (Makarenko et al., 2002) use a similar idea including the uncertainty in the localization as part of the utility function in the assignment of desti- nations to robots. These 3 techniques are based on the estimation of landmarks and they try to prevent that the uncertainty in the pose of the robots grows, by means of keeping always well estimated landmarks in the field of view. (Sim et al., 2004) recover the certainty over the pose of the robots during the exploration using a parametric curve trajectory and including returning to explored zones when the uncertainty in the pose of the robot is too high. (Stachniss et al., 2005) reduce the uncertainty by actively closing loops with previously explored areas. They create a topological map of the environment and look for opportunities for closing loops in it. As we can see, there are two main approaches to the problem of localization during the exploration: to take the uncertainty in the pose of the robots into account when choosing the movements for the robots or to explore and return later to previously explored zones when the uncertainty is large.

In this paper, a potential field based SPLAM technique is described. It is based on the potential field generated by several basic behaviours designed to rapidly explore the environment. It also considers returning to previously explored zones when needed.

\section{BEHAVIOUR-BASED EXPLORATION ALGORITHM}

In typical environments we can find a set of highly distinctive elements that can be easily extracted with the sensors of a robot. These elements are typically called landmarks. In our application, we assume that the robots are able to detect a set of distinctive $3 \mathrm{D}$ visual landmarks and they are able to obtain relative measurements to them using stereo cameras. These landmarks can be extracted as interest points found in the images of the environment (Mozos et al., 2007). The robot team is able to build a map with a vision-based technique consisting on a particle filter approach to the SLAM problem, known as FastSLAM (Gil et al., 2007).

Landmark based maps do not represent the free or occupied areas in the environment. This is the reason why we make use of a grid map to represent free and occupied cells detected using the information of the sonar. In addition, all the cells have a numerical value associated that indicates their degree of exploration, which is increased each time it falls into the field of view of the robot, until it reaches a limit value when the cell is considered to be fully explored. A cell with an exploration degree of zero is considered unexplored. We define the frontier cells as explored cells that lie next to an unexplored cell that do not 
Table 1: Forces defined for each behavior

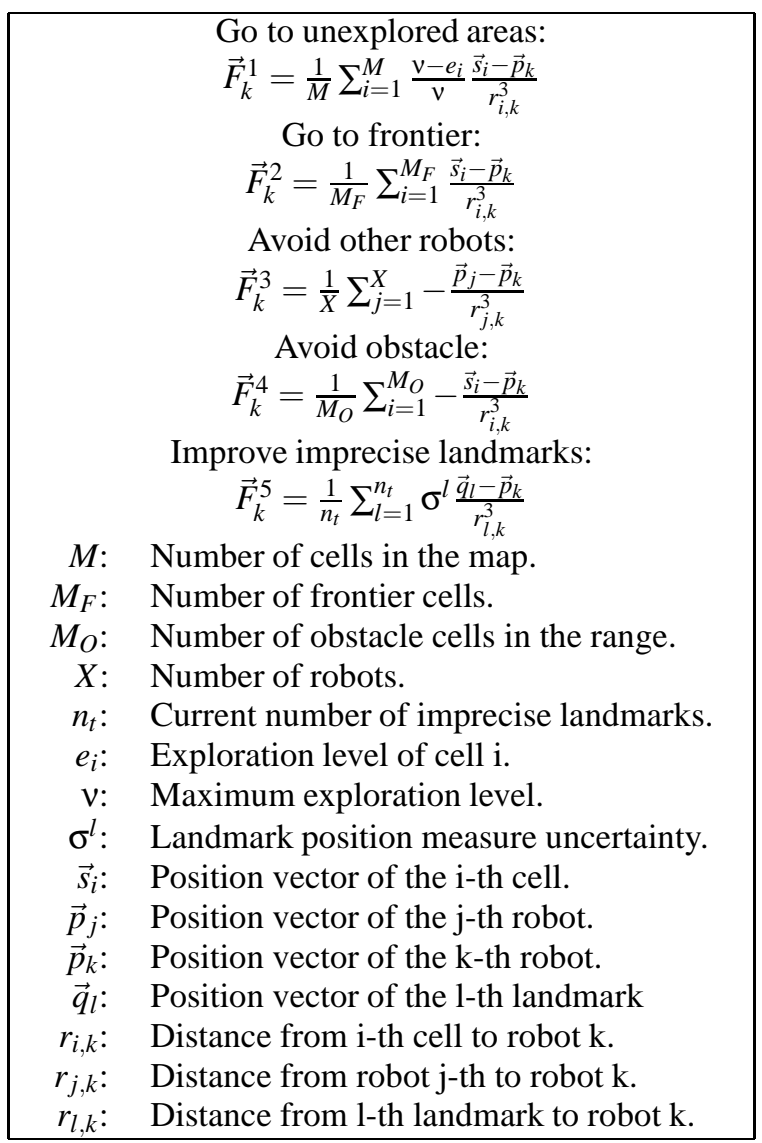

belong to an obstacle.

Our approach to the problem of multi-robot exploration consists of five basic behaviours whose composition results in the trajectory of each robot in the environment:

Go to unexplored areas: Each cell attracts each robot with a force that depends on the degree of exploration of the cell.

Go to frontier: This behaviour attracts the robots to frontier cells since these are the cells that give way to areas of interest.

Avoid other robots: This behaviour results in a repulsive force between robots that normally allows to spread the robots around the environment.

Avoid obstacle: Each cell within a specific range that is identified as belonging to an obstacle, applies a repulsive effort over every robot. This range allows to easily adjust the system.

Improve imprecise landmarks: This behaviour tries to improve the quality of the exploration of those areas where some landmarks have been extracted but whose accuracy is not high enough.

Table 1 shows how the forces are calculated for

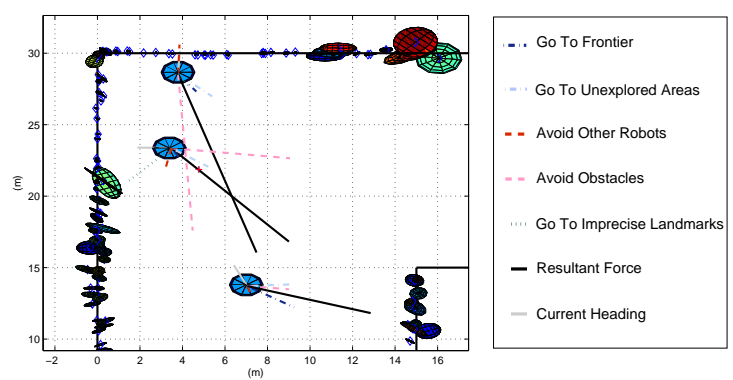

Figure 1: Weighted outputs of the behaviours and resultant force in an exploring situation. Also, the landmarks that have been detected until that moment are shown.

each behaviour. This way, the resulting force of the combination of those five behaviours on each robot constitutes a vector that indicates the trajectory of the robot to optimize the exploration process as follows:

$$
\vec{F}_{k}^{A}=k_{1} \vec{F}_{k}^{1}+k_{2} \vec{F}_{k}^{2}+k_{3} \vec{F}_{k}^{3}+k_{4} \vec{F}_{k}^{4}+k_{5} \vec{F}_{k}^{5} .
$$

The composition of the behaviours is carried out taking into account a set of weights $k_{i}$ whose value is deduced experimentally. Fig. 1 shows the bird's eye view of an exploring situation with three robots.

Potential field methods have a main disadvantage: when exploring complex environments, a robot may be trapped at local minima in the potential field and may not move, thus stopping the exploration process. To solve this problem, we assume that we are able to detect the situation in which the robot is trapped at a local minimum. In this case, a new state is triggered that enables the robot to escape from the local minimum by planning a path to the nearest frontier cell.

\section{INTEGRATED EXPLORATION}

As an unknown environment is explored the uncertainty in the localization of the robot grows. When the uncertainty over the pose of the robots is high, it is difficult to generate a correct map, and thus the exploration process is inefficient. If the error in the localization is very high, some frontiers and obstacles could be added to the grid map erroneously and some zones could remain unexplored. The perceptions of the robots in a given moment can be in conflict with past perceptions or with perceptions of other robots because of a deficient localization.

Figure 2 shows an example of an extremely deficient exploration caused by a large error in the localization. It can be observed how the wrong location of some obstacles obstructs the corridor and part 

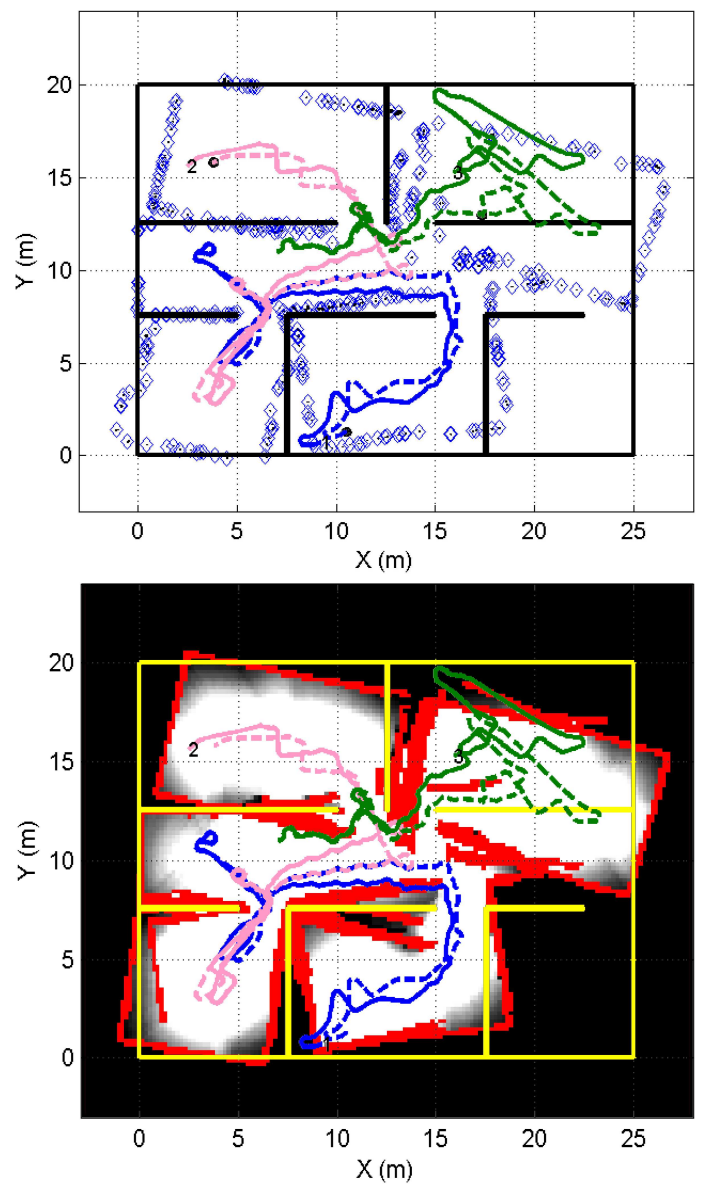

Figure 2: The upper figure shows the visual landmark map created in an exploration with deficient localization. The trajectories performed by the the robots are indicated in continuous lines and the estimated trajectories in discontinuous lines. The landmarks detected are marked in their estimated position. The bottom figure shows the grip map generated for this situation. The grade of exploration is indicated in gray levels and the obstacles detected in red color. Real obstacle positions are marked in yellow.

of the environment remain unexplored. The error in the map of landmarks created is considerably high. All the landmarks in the map should appear over the walls but they are situated erroneously. These are the reasons we introduce new techniques to improve the localization.

The SLAM method we use in our experiments is commonly known as FastSLAM (Gil et al., 2007). It consists of a particle filter, each particle having an estimation for the path of each robot and an estimation of a set of landmarks conditioned to the path. We can measure the uncertainty on the localization of a robot by considering the dispersion for all the particles in the position of the robot. When travelling through un- known terrain, the dispersion of the particles usually increases since each is well localized in his own local map. Since we are using a finite number of particles to represent the pose of the robots, this representation gets worse when the uncertainty is too high. In this case we consider returning to previously explored areas to reduce this uncertainty. This idea has been employed by many authors (Feder et al., 1999; Bourgoult et al., 2002; Makarenko et al., 2002; Sim et al., 2004; Stachniss et al., 2005). Avoiding large periods of time with a high dispersion is a good technique to avoid the accumulation of error in the global localization and an accurate map can be obtained. Thus, our strategy is to return to positions with low dispersion when the dispersion in the pose of the robot grows. This solution produces a better estimation of the map and the robot's path.

We denote the model explained in Section 3 as the Exploration State (StateA). Besides, we introduce an Active Localization State (StateB). The Exploration State allows exploring new areas of the map meanwhile the robots are well localized. The Active Localization State intends to lead the robots to previously explored areas when they have a relatively high uncertainty associated, thus improving their localization. The transition between both states is made according to a hysteretic model with two transition thresholds that are compared with the dispersion in the pose of the robot.

In the Active Localization State, the control action of the robot is the composition of Avoid Obstacle, already presented, and a new behaviour Go to Accurate Landmarks. This new behavior aims at localizing the robot returning to previously explored landmarks.

Go to Accurate Landmarks: This behaviour tries to improve the estimation of the position of the robot, driving it to landmarks whose position has a robust estimation. Given a landmark, its position is calculated for each particle and a measure of its dispersion $\varepsilon^{l}$ is calculated using the correspondent landmarks for the different particles. The correspondence is done considering an unique visual descriptor for each landmark. Each accurate landmark attracts the robot with a force inversely proportional to the distance:

$$
\vec{F}_{k}^{6}=\frac{1}{n} \sum_{l=1}^{n} \frac{1}{\varepsilon^{l}} \frac{\vec{q}_{l}-\vec{p}_{k}}{r_{l, k}^{2}} .
$$

being $n$ the current number of landmarks in the map, $\vec{q}_{l}$ is the position of the 1-th landmark, $\vec{p}_{k}$ is the position of the robot $k$ and $r_{l, k}$ is the euclidean distance between both positions. Then the trajectory to follow is pointed by the vector:

$$
\vec{F}_{k}^{B}=k_{4} \vec{F}_{k}^{4}+k_{6} \vec{F}_{k}^{6}
$$




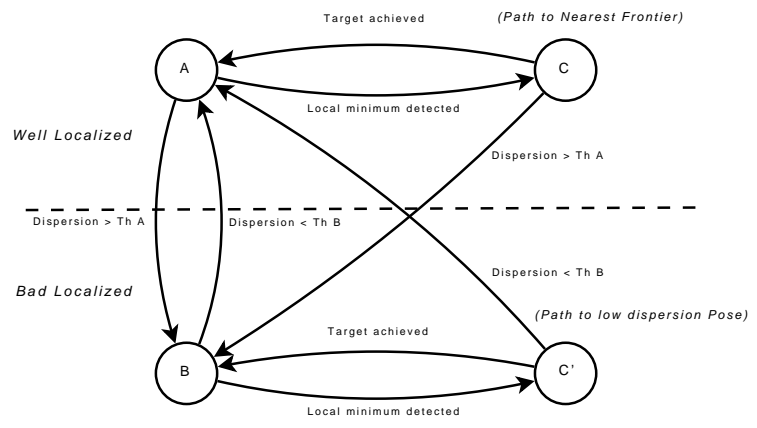

Figure 3: State transition diagram

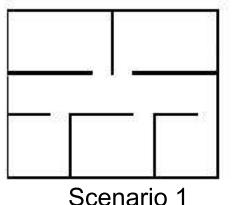

Scenario 1

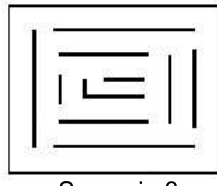

Scenario 3

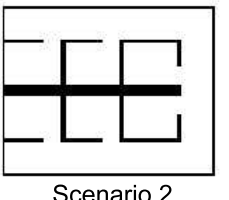

Scenario 2

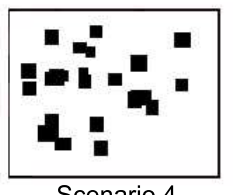

Scenario 4

Figure 4: Scenarios

where the weights are deduced experimentally.

As stated before, the local minima in the potential field can block the exploration process. In these cases, we plan a path to the nearest frontier cell. This solution directs the robots to unknown areas and thus is only a good solution in the State A. In StateB, local minima are also likely to appear. In this case, we plan a path to the last past position in the trajectory of the robot where the dispersion is low.

Figure 3 shows the state diagram for a robot. We can distinguish two zones of operation: when the robot is well localized and when it is not. When it is well localized, it explores the environment by following the State A combination of behaviors. If it finds a local minimum during the exploration it plans a path (State $C$ ) to the nearest frontier cell. When it arrives to this cell it returns to the State A. If the dispersion on the robot position in the particle filter is over a given threshold the robot is considered to be bad localized and it switches to the Active Localization State (B). If it finds a local minimum being in this state, it plans a path $\left(C^{\prime}\right)$ to a past position in the trajectory with low dispersion. When the dispersion decreases below a threshold the robot returns to the Exploration State (A).

\section{EXPERIMENTS AND RESULTS}

In this section, we analyze simulation results of the method proposed in this paper. The proposal is tested in presence of uncertainty in the robots localization to show the improvement in the quality of the maps generated and in the estimated path using the techniques proposed to return to previously explored areas.

The scenarios chosen to test the method are shown in Figure 4. Scenarios that represent hypothetical real places like Scenario 1 or Scenario 2 were chosen at the same time that other artificial scenarios as for example Scenario 3 or a completely random scene as

\section{Scenario 4.}

The method proposed is tested with and without considering the uncertainty in the position of the robots. Besides, it is compared with a pure path planning approach where the robots always plan a path to the nearest frontier cell. The mean error per robot in the estimated trajectories, the exploration time, as well as the error in the map of landmarks are analyzed.

The results of the simulation are shown in Figure 5. On Scenario 1 and Scenario 4, the error on the estimated path and in the map is smaller with the proposed integrated exploration approach than when not returning to previously explored zones. This two scenarios have large free spaces. When the robots travel in these zones the measurement of the landmarks is difficult as they are far away. This makes the difference between including the Go To Accurate Landmarks behaviour or not. This large periods of time with bad localization increases the global localization error when not considering the dispersion to try to relocalize the robot.

For Scenario 2 and Scenario 3 we do not observe an improvement. Note that the global localization error depends on the form of trajectories and the exploration time that depend on the structure of the environments which is unknown. If the measurements are good enough this other factors affects in a random way and in average no difference is observed between the methods.

The exploration time always increases because this method does not always guide the robots to the direction of the maximal information gain as it looks also for the localization. As a conclusion, we think that a method that only tries to minimize the exploration time produces normally inaccurate maps useless for navigation. We consider that taking into account the requisites of SLAM while exploring the environment allows to obtain more precise maps. 


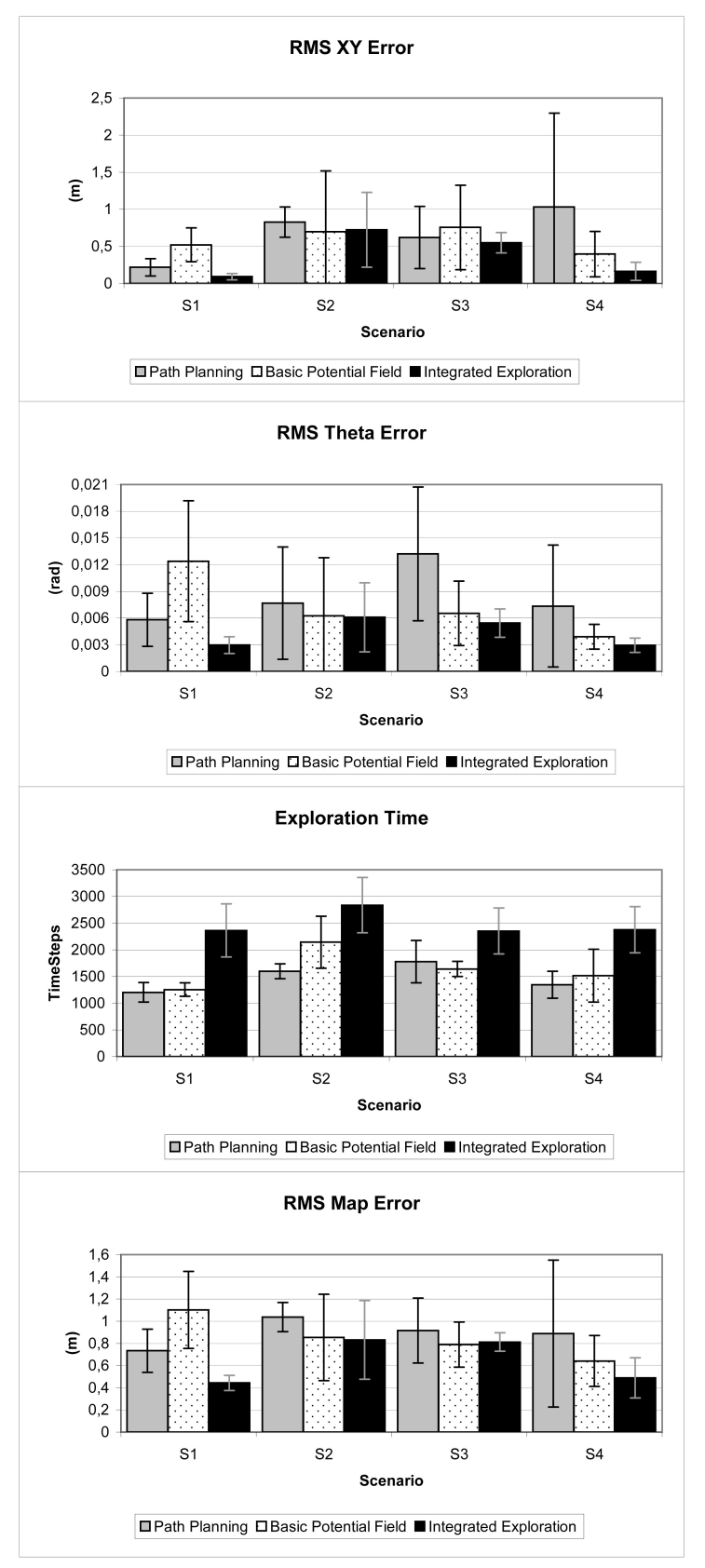

Figure 5: Results with uncertainty in localization: Path error on $\mathrm{x}, \mathrm{y}$ and orientation (1st and 2nd graphs), exploration time (3rd graph) and map error (4th graph).

\section{CONCLUSIONS AND FUTURE WORK}

In this paper a method for multi-robot cooperative exploration has been presented. The method is based on the computation of a set of behaviours designed so that we simultaneously consider the necessity of rapidly exploring the whole environment and the requisite to build an accurate map. In this sense, the method directs the robots to return to previously explored places when the uncertainty on the location becomes significant, and this fact improves the quality of the resulting map. Several simulation results demonstrate the validity of the approach. On scenarios with large free space where there is a lack of good measures of the landmarks the accuracy of the map improves considerably.

As future works, we consider the extension of the approach in dynamic environments, adding techniques to learn automatically the multiple settings of the system. New behaviors that avoid the dispersion in the localization by trying to keep accurate landmarks in the field of view will be added. Furthermore, behaviors of attraction between robots will be incorporated in order to improve the localization since the observation of one robot by other member in the team may improve its localization. Semi-operated models that integrate the commands expressed by a human operator in the exploration task will also be studied, where these commands would be taken as an advice.

\section{ACKNOWLEDGMENTS}

This work has been supported by the Spanish Government (Ministerio de Educación y Ciencia). Project: 'Sistemas de percepción visual móvil y cooperativo como soporte para la realización de tareas con redes de robots'. Ref.: DPI2007-61197.

\section{REFERENCES}

Arkin, R. and Diaz, J. (2002). Line-of-sight constrained exploration for reactive multiagent robotic teams. 7 th International Workshop on Advanced Motion Control, AMC'02, Maribor, Slovenia.

Bourgoult, F., Makarenko, A., Williams, S., Grocholsky, B., and Durrant-Whyte, F. (2002). Information based adaptive robotic exploration. In Proc. of the IEEE/RSJ Int. Conf. on Intelligent Robots and Systems (IROS), Lausanne, Switzerland.

Burgard, W., Moors, M., Stachniss, C., and Schneider, F. (2005). Coordinated multi-robot exploration. IEEE Transactions on Robotics, Vol. 21 No3 pp 376-386, June.

Cao, Y., Fukunaga, A. S., Kahng, A. B., and Meng, F. (1995). Cooperative mobile robotics: Antecedents and directions. In IEEE/TSJ International Conference on Intelligent Robots and Systems, Yokohama, Japan.

Feder, H., Leonard, J., and Smith, C. (1999). Adaptive mobile robot navigation and mapping. Int. Journal of Robotics Research, 18(7). 
Gil, A., Reinoso, O., Payá, L., and Ballesta, M. (2007). Influencia de los parámetros de un filtro de partículas en la solución al problema de slam. Accepted for publication in the IEEE Latin America.

Lau, H. (2003). Behavioural approach for multi-robot exploration. Australasian Conference on Robotics and Automation (ACRA 2003), Brisbane, December.

Makarenko, A., Williams, S., Bourgoult, F., and DurrantWhyte, F. (2002). An experiment in integrated exploration. In Proc. of the IEEE/RSJ Int. Conf. on Intelligent Robots and Systems (IROS), Lausanne, Switzerland.

Mozos, O. M., Gil, A., Ballesta, M., and Reinoso, O. (2007). Interest point detectors for visual slam. Proc. of the Conference of the Spanish Association for Artificial Intelligence (CAEPIA), Salamanca, Spain, November.

Sim, R., Dudek, G., and Roy, N. (2004). Online control policy optimization for minimizing map uncertainty during exploration. In Proc. of the IEEE Int. Conf. on Robotics and Automation (ICRA), New Orleans, LA, USA.

Simmons, R., Apfelbaum, D., Burgard, W., Fox, D., Moors, M., Thrun, S., and Younes, H. (2000). Coordination for multi-robot exploration and mapping. In Proceedings of the AAAI National Conference on Artificial Intelligence, Austin, TX.

Stachniss, C., Haehnel, D., Burgard, W., and Grisetti, G. (2005). Actively closing loops in grid-based fastslam. information. Advanced Robotics - The Int. Journal of the Robotics Society of Japan (RSJ), Volume 19, number 10, pages 1059-1080.

Yamauchi, B. (1997). A frontier based approach for autonomous exploration. IEEE International Symposium on Computational Intelligence in Robotics and Automation, Monterey, CA, July 10-11.

Zlot, R., Stentz, A., Dias, M. B., and Thayer, S. (2002). Multi-robot exploration controlled by a market economy. Proceedings of the IEEE International Conference on Robotics and Automation. 